\title{
Vestibular Rehabilitation to the Patients With Vestibular Migraine and Vestibular Neuritis
}

Fumiyuki Goto ( $\square$ amifumi@gmail.com )

Department of Otolaryngology, Tokai university, kanagawa, Japan

Nagisa Sugaya

Yokohama City University: Yokohama Shiritsu Daigaku

Kenji Okami

Tokai University Tokyo Hospital: Tokai Daigaku Igakubu Fuzoku Tokyo Byoin

Masahiro lida

Tokai University Tokyo Hospital: Tokai Daigaku Igakubu Fuzoku Tokyo Byoin

\section{Research}

Keywords: vestibular migraine (VM), Profile of Mood States (POMS), physical component score (PCS)

Posted Date: January 18th, 2021

DOI: https://doi.org/10.21203/rs.3.rs-145328/v1

License: () (1) This work is licensed under a Creative Commons Attribution 4.0 International License. Read Full License 


\section{Abstract}

\section{Background}

It is not yet clarified the effect of vestibular rehabilitation on patients with vestibular migraine (VM).

\section{Aims/Objectives}

In this study, we aimed to compare the effect of vestibular rehabilitation on patients with VM and those in the chronic stage of vestibular neuritis (VN).

\section{Material and Methods}

A total of 26 patients with VM and 31 patients in the chronic stage of VN who were treated. All patients underwent an in-hospitalized vestibular rehabilitation program. A variety of data including the Dizziness Handicap Inventory (DHI), POMS, and posturography were compared.

\section{Results}

Before treatment, there was no significant difference in the parameters between the two groups, except for a higher confusion score of Profile of Mood States (POMS) in the VM group. In both groups, the DHI score significantly improved. In the VM group, the confusion score of POMS, physical component score (PCS) of SF-8, and some parameters in posturography significantly improved. In the VN group, anxiety, depression, and PCS of SF-8 significantly improved.

\section{Conclusions and Significance}

The higher confusion score of POMS in patients with VM reflects the known increased comorbidity risk of psychiatric disorders. In both groups, in-hospitalized vestibular rehabilitation was effective in alleviating the subjective and objective parameters of vestibular symptoms within a month.

\section{Introduction}

An association between vestibular symptoms, including dizziness and vertigo, and headache, has been reported in several studies [1]. The diagnostic criteria for "vestibular migraine" (VM) are described in the International Classification of Headache Disorders (ICHD), third edition, beta version[2]. Patients with chronic dizziness often complain of not only physical complaints but also mental issues. There are reports on the high rate of psychiatric comorbidity in patients with chronic dizziness, including VM $[3,4]$, which may contribute to the treatment outcomes.

There is moderate to strong evidence that vestibular rehabilitation is safe and effective for managing unilateral peripheral vestibular dysfunction, based on several high-quality randomized controlled trials [5]. However, there is insufficient evidence for vestibular rehabilitation in patients with VM. Sugaya et al. reported the effect of vestibular rehabilitation in a patient with VM [6]. However, in their study, the effect of rehabilitation was not compared with a specific group of patients with dizziness but was compared with patients with tension-type headache and various states of dizziness. It is clinically useful to compare the effect of vestibular rehabilitation between VM and other vestibular diseases, such as vestibular neuritis (VN), where the efficacy of vestibular rehabilitation has been sufficiently demonstrated [7]. For analysis of the patient's mood, they only evaluated anxiety and depression using the Hospital Anxiety and Depression Scale. Headache is reported to be associated with various emotions [8]; thus, multidimensional emotions in VM should be investigated. Additionally, the health-related quality of life (QOL) is reportedly lower in participants with VM than in controls without dizziness [9]. Improvement of the health-related QOL in VM is of clinical importance.

This study aimed to compare the characteristics and effects of vestibular rehabilitation on patients with VM and VN. We tried to analyze not only the dizziness handicap but also multidimensional emotions and the mental and physical health-related QOL in the patients.

\section{Materials And Methods}

Patients with a chief complaint of dizziness who visited the Department of Otorhinolaryngology at the National Tokyo Medical Center were included in this study. A retrospective chart review was conducted. A total of 26 patients ( 4 males, 22 females; average age $51.2 \pm 18.6$ years) with VM and 31 patients ( 10 males, 21 females, $57.1 \pm 12.4$ years) in the chronic stage of VN were treated at our institute from 2015 to 2017 . Participants complained of persistent dizziness even after conventional treatment, which included (1) drug therapy with betahistine $36 \mathrm{mg}$ daily for the initial 2-4 weeks, (2) lifestyle counseling regarding exercise and daily walking, and (3) attaining adequate sleep and stress reduction. We recruited participants who met the above-mentioned criteria if (1) the patient was $\geq 20$ years of age, (2) the dizziness had persisted for at least 3 months despite conventional therapy in the outpatient clinic, (3) the patient wished to have intensive, inpatient therapy for the persistent dizziness, and (4) the patient was literate. The exclusion criteria were as follows: (1) a diagnosis of dizziness due to a cerebrovascular disorder, (2) medical contraindications for the necessary head movements during vestibular rehabilitation (e.g., severe cervical disorder), (3) serious comorbidity (for instance, a life-threatening condition, severe cognitive impairment, or severe psychiatric disorder), (4) central nervous system disease, or (5) bilateral vestibular deficit. The diagnosis of VM was based on the ICHD, third edition, beta version [2] and VN was based on the diagnostic criteria of the Japan Equilibrium Research Association [10]. Patients with VN complained of dizziness for at least 3 months after the diagnosis of VN.

This study was approved by the ethical committee of the National Tokyo Medical Center (R12-009) and was performed in accordance with the ethical standards laid down in the 1964 Declaration of Helsinki and its later amendments. 
Dizziness Handicap Inventory

The Dizziness Handicap Inventory (DHI) [11] is a standard questionnaire that quantitatively evaluates the degree of handicap in the daily lives of patients with vestibular disorders and consists of 25 questions. The total score ranges from 0 (no disability) to 100 (severe disability).

State-Trait Anxiety Inventory

The State-Trait Anxiety Inventory (STAI) [12] is a self-reported questionnaire consisting of 40 questions, comprising a state anxiety subscale with 20 items and a trait anxiety subscale with 20 items. Each item of the STAI assesses how respondents "generally" feel regarding each statement (e.g., "I feel at ease") on a scale of 1 (almost never) to 4 (almost always).

Self-Rating Depression Scale

The Self-Rating Depression Scale (SDS), designed by Zung in 1965 [13], is generally considered a reliable instrument for measuring depressive symptoms in primary care. It is a self-reported, 20-item questionnaire. Item responses are rated from 1 to 4 , with higher scores corresponding to more frequent symptoms.

Profile of Mood States (POMS)

The POMS is a psychological rating scale used to assess transient, distinct mood states. The POMS measures six different dimensions of mood swings over a period of time. These include tension or anxiety, anger or hostility, vigor or activity, fatigue or inertia, depression or dejection, and confusion or bewilderment. A five-point scale ranging from "not at all" to "extremely" is administered by experimenters to patients for assessing their mood state.

Japanese version of the Medical Outcomes Study 8-items Short Form Health Survey

Health-related QOL was evaluated using the Japanese version of the Medical Outcomes Study 8-items Short Form Health Survey (SF-8) questionnaire, the validity and reliability of which have already been confirmed [14]. SF-8 comprises eight items - physical functioning, role limitation due to physical problems, body pain, general health, vitality, social functioning, role limitation due to emotional problems, and mental health. The physical health component summary score (PCS) and mental health component summary score (MCS) were measured using the norm-based scoring method, which is based on a large-scale population study conducted in Japan [15]. Higher scores on these subscales indicate better health-related QOL.

Measurement of the gravity center fluctuation

The measurement of the gravity center fluctuation for objective assessment of the dizziness severity was performed using a stabilometer (G-5000, Anima Corp., Tokyo, Japan), and the total length of path (LNG) and environmental area (ENV) during eye-opening/closing.

\section{Intervention}

Patients were hospitalized for 5 days in groups of 8-10 individuals; the groups were then taught how to self-perform the 30-min vestibular rehabilitation program [16], which comprised repeatedly performing the vestibulo-ocular reflex (VOR) and vestibulo-spinal reflex (VSR). The VOR training included seven exercises: (1) quick horizontal eye movement, (2) quick vertical eye movement, (3) eye tracking the horizontal direction, (4) eye tracking the vertical direction, (5) horizontal head shaking with gazing at a fixed target, (6) vertical head shaking with gazing at a fixed target, and (7) oblique head tilting with gazing at a fixed target. Each eye or head movement was repeated 20 times. The VSR training consisted of eight static and five dynamic exercises. The eight static exercises were: (1) standing up and sitting down with the eyes open, repeated thrice; (2) standing up and sitting down with the eyes closed, repeated thrice; (3) standing with the eyes closed and feet apart for $20 \mathrm{~s}$; (4) standing with the eyes closed and feet together for $20 \mathrm{~s}$; (5) standing in a tandem posture with the right foot in the front for $20 \mathrm{~s}$; (6) standing in a tandem posture with the left foot in the front for $20 \mathrm{~s}$; (7) standing on the right foot for $20 \mathrm{~s}$; and (8) standing on the left foot for $20 \mathrm{~s}$. The five dynamic exercises were: (1) $180^{\circ}$ turn to the left, repeated thrice; (2) $180^{\circ}$ turn to the right, repeated thrice; (3) walking in a tandem posture for $10 \mathrm{~m}$; (4) walking with horizontal head shaking for $10 \mathrm{~m}$; and (5) walking with vertical head shaking for $10 \mathrm{~m}$. During the training, patients performed these exercises thrice a day under the supervision of a physician. After 5 days, all patients learned to perform the exercises. The patients were then instructed to continue performing the vestibular rehabilitation program thrice a day after discharge.

\section{Procedure}

After the participants provided written informed consent, they were evaluated on the day of hospitalization (time 1), as well as a month later (time 2), using the questionnaires mentioned previously. Static posturography was also performed. All drugs that could affect dizziness, including vestibular suppressants, were discontinued soon after the introduction of vestibular rehabilitation.

\section{Statistical analysis}

Data analysis was performed using the SPSS 25.0 software (IBM Corp, NY, USA). A two-way repeated measures analysis of variance (ANOVA) was performed to analyze the effects of group and time on all outcomes. The t-test was used for group comparisons of age. A P-value $<0.05$ was considered significant.

\section{Results}

Descriptive results 
Forty-nine patients ( 12 males and 37 females, mean age $=54.1 \pm 16.3$ years) remained in the analysis. The participants in this study comprised 25 patients with VM and 24 patients with VN. There was no significant difference in age between the groups $(\mathrm{t}[41.6]=1.3, \mathrm{p}=0.21)$. Some patients did not provide any data regarding STAI responses (valid data: $N=48$ ), SDS responses (valid data: $N=47$ ), POMS responses (valid data: $N=47$ ), SF-8 responses (valid data: $N=$ 46), and LNG and ENV during eye opening and closing (valid data: $N=46$ ). In cases where the participant fell, measurement of their center of gravity fluctuation was treated as missing data.

Two-way ANOVA results

Table 1 shows the comparison between groups and time points for all variables using two-way repeated measures ANOVA. There were significant main effects of group on the trait scale of STAI and the SDS score $(\mathrm{VM}>\mathrm{VN})$ and significant main effects of time on the DHI score, state and trait scores of STAI, SDS score, trait-anxiety, depression, anger-hostility, confusion, and total mood disturbance scores of POMS, the PCS and MCS scores of SF-8, LNG during eye closing, and ENV during eye closing (SF-8: time 1 < time 2, other variables: time 1 > time 2). Regarding the confusion score of POMS, there was a significant interaction between group and time. The post hoc test showed that the confusion score at time 1 in the VM group was significantly higher than that in the VN group and that the confusion score at time 2 was significantly lower than that at time 1 only in the VM group.

\section{Discussion}

In this study, we successfully indicated that the group undergoing vestibular rehabilitation showed improvement almost all parameters of dizziness, emotions, and QOL for both VM and VN. The improvement of physical and psychological symptoms in patients with VM supports our previous results [6]. Additionally, our provides new findings on the effect on anger, confusion, or QOL, which were not investigated in a previous study [6]. Migraine or VM is closely associated with psychosocial stress and should be assessed using both physical and psychological measurements.

The mechanism of efficacy of vestibular rehabilitation in patients with VM can be explained by the physiological background of the association between vestibular function and headache. Some studies have proposed that the mechanism underlying vestibular dysfunction related to migraine is a parallel activation of the vestibular and cranial nociceptive pathways [8]. Nociceptive and vestibular afferents with neurochemical similarities, including the expression of serotonin, capsaicin, and purinergic receptors[8], converge in brainstem structures such as the parabrachial nucleus, raphe nuclei, and locus coeruleus, and these structures play an important role in modulating the sensitivity of pain pathways [8]. Thus, vestibular symptoms may have a biologically close association with headaches.

In this study, the "confusion" score, a subscale of the POMS, indicated the most prominent difference between patients with VM and patients with VN regarding the efficacy of vestibular rehabilitation. The migraineurs reported significantly greater scores on the confusion-bewilderment subscale of the POMS [17]. We also found that patients with VM had a higher score of confusion of POMS at baseline. Additionally, the confusion score improved with vestibular rehabilitation only in patients with VM. Anxiety in patients with VM was significantly higher than that in those with VN in STAI but not in POMS. This discrepancy may be due to a similar level of anxiety in VM and VN. Patients with VN had a high level of anxiety [18], comparable with that of patients with VM.

Our study had several limitations. First, the number of patients with both VM and VN was small. In the future, we will conduct a similar study with a larger sample and compare sexes and age groups or apply appropriate regression analyses. Second, the study was not prospective but a retrospective chart review.

Third, the study periods were relatively short, being only a month. In the future, we will conduct a prospective study with long-term follow-up. Fourth, this study lacked a control group. The effect of vestibular rehabilitation is worth investigating using a randomized controlled protocol in order to obtain more convincing evidence.

\section{Conclusions}

Vestibular rehabilitation contributed to an improvement in vertigo along with dizziness and psychological factors in both patients with VM and VN.

\section{Declarations}

\section{Author Contributions}

FG drafted the manuscript. MA collected the data. FG organized the research, and NS analyzed the data and checked the manuscript.

Declaration of interest

The authors declare that the research was conducted in the absence of any commercial or financial relationships that could be construed as a potential conflict of interest.

Funding

Part of this study was supported by a grant from the Mental Health Okamoto Memorial Foundation 2016.

\section{References}

[1] Dieterich M, Brandt T: Episodic vertigo related to migraine (90 cases): vestibular migraine? J Neurol 1999;246:883-92. 
[2] Lempert T, Olesen J, Furman J, et al.: [Vestibular migraine: diagnostic criteria: consensus document of the Barany Society and the International Headache Society]. Nervenarzt 2013;84:511-6.

[3] Peluso ET, Quintana MI, Gananca FF: Anxiety and depressive disorders in elderly with chronic dizziness of vestibular origin. Braz J Otorhinolaryngol 2016;82:209-14.

[4] Eggers SD, Neff BA, Shepard NT, et al.: Comorbidities in vestibular migraine. J Vestib Res 2014;24:387-95.

[5] McDonnell MN, Hillier SL: Vestibular rehabilitation for unilateral peripheral vestibular dysfunction. Cochrane Database Syst Rev 2015;1:CD005397.

[6] Sugaya N, Arai M, Goto F: Is the Headache in Patients with Vestibular Migraine Attenuated by Vestibular Rehabilitation? Front Neurol 2017;8:124.

[7] Tokle G, Morkved S, Brathen G, et al.: Efficacy of Vestibular Rehabilitation Following Acute Vestibular Neuritis: A Randomized Controlled Trial. Otol Neurotol 2020;41:78-85.

[8] Perozzo P, Savi L, Castelli L, et al.: Anger and emotional distress in patients with migraine and tension-type headache. J Headache Pain 2005;6:392-9.

[9] Neuhauser HK, Radtke A, von Brevern M, et al.: Migrainous vertigo: prevalence and impact on quality of life. Neurology 2006;67:1028-33.

[10] Komatsuzaki A, Futaki T, Harada Y: [Documents for diagnostic standardization of dizziness 1987]in Japanese. Equilibrium Research 1988;47:245-73.

[11] Goto F, Nomura K, Taka F, et al.: Analysis of Factors Affecting the Outcomes of In-hospitalized Vestibular Rehabilitation in Patients With Intractable Dizziness. Otol Neurotol 2017;38:368-72.

[12] Spielberger CD: State-Trait Anxiety Inventory: Bibliography (2nd ed) Palo Alto, CA, Consulting Psychologists Press., 1989, vol 17, pp 101-6.

[13] Zung WW, Richards CB, Short MJ: Self-rating depression scale in an outpatient clinic. Further validation of the SDS. Arch Gen Psychiatry 1965;13:50815 .

[14] Ware JE, Kosinki M, Dewey J, et al.: How to Score and Interpret Single-Item Health Status Measures: A Manual for Users of the SF-8 Health Survey. . Boston, QualityMetric Inc., 2001.

[15] Fukuhara S, Suzukamo Y: Manual of the SF-8 Japanese Version. Kyoto, Institute for Health Outcome and Process Evaluation Research, 2004.

[16] Sugaya N, Arai M, Goto F: Changes in cognitive function in patients with intractable dizziness following vestibular rehabilitation. Sci Rep 2018;8:9984.

[17] Kozak HH, Boysan M, Uca AU, et al.: Sleep quality, morningness-eveningness preference, mood profile, and levels of serum melatonin in migraine patients: a case-control study. Acta Neurol Belg 2017;117:111-9.

[18] Best C, Eckhardt-Henn A, Diener G, et al.: Interaction of somatoform and vestibular disorders. J Neurol Neurosurg Psychiatry 2006;77:658-64.

\section{Tables}

Table 1. Comparison of all variables between groups and time points (two-way repeated measures analysis of variance). 


\begin{tabular}{|c|c|c|c|c|c|c|c|c|c|c|c|c|c|c|}
\hline & \multicolumn{4}{|c|}{ VM (Mean $\pm S D)$} & \multicolumn{4}{|c|}{ VN (Mean $\pm S D)$} & \multicolumn{2}{|c|}{$\begin{array}{l}\text { Main effect } \\
\text { of group }\end{array}$} & \multicolumn{2}{|c|}{$\begin{array}{l}\text { Main effect of } \\
\text { time }\end{array}$} & \multicolumn{2}{|c|}{ Interactic } \\
\hline & \multicolumn{2}{|l|}{ Time 1} & \multicolumn{2}{|l|}{ Time 2} & \multicolumn{2}{|c|}{ Time 1} & \multicolumn{2}{|l|}{ Time 2} & \multirow{2}{*}{$\begin{array}{l}\mathrm{F} \\
0.0\end{array}$} & \multirow{2}{*}{$\begin{array}{l}p \\
0.93\end{array}$} & \multirow{2}{*}{$\begin{array}{l}F \\
70.2\end{array}$} & \multirow{2}{*}{$\begin{array}{l}p \\
<0.0001\end{array}$} & \multirow{2}{*}{$\begin{array}{l}\mathrm{F} \\
1.5\end{array}$} & \multirow{2}{*}{$\begin{array}{l}\mathrm{p} \\
0 .:\end{array}$} \\
\hline DHI & 50.1 & \pm 20.5 & 30.1 & \pm 26.0 & 53.1 & \pm 16.3 & 26.2 & \pm 16.7 & & & & & & \\
\hline \multicolumn{15}{|l|}{ STAI } \\
\hline State & 49.7 & \pm 8.7 & 45.5 & \pm 9.8 & 47.6 & \pm 12.4 & 41.2 & \pm 8.3 & 1.7 & 0.19 & 12.5 & 0.001 & 0.6 & 0. \\
\hline Trait & 52.7 & \pm 11.2 & 47.9 & \pm 11.7 & 44.8 & \pm 9.7 & 39.0 & \pm 9.2 & 10.1 & 0.003 & 12.5 & 0.001 & 0.1 & 0. \\
\hline SDS & 48.6 & \pm 11.6 & 43.8 & \pm 10.4 & 43.5 & \pm 7.3 & 38.5 & \pm 8.1 & 4.4 & 0.04 & 14.0 & 0.001 & 0.0 & 0.1 \\
\hline \multicolumn{15}{|l|}{ POMS } \\
\hline $\begin{array}{l}\text { Tension- } \\
\text { Anxiety }\end{array}$ & 8.5 & \pm 5.2 & 6.0 & \pm 4.2 & 6.8 & \pm 3.8 & 5.0 & \pm 3.6 & 1.5 & 0.22 & 11.8 & 0.001 & 0.3 & 0.1 \\
\hline Depression & 6.4 & \pm 4.3 & 4.8 & \pm 3.6 & 4.6 & \pm 3.5 & 3.5 & \pm 4.0 & 2.4 & 0.13 & 8.2 & 0.006 & 0.2 & 0.1 \\
\hline $\begin{array}{l}\text { Anger- } \\
\text { Hostility }\end{array}$ & 6.2 & \pm 4.8 & 5.4 & \pm 3.9 & 5.2 & \pm 4.9 & 3.5 & \pm 2.7 & 1.7 & 0.20 & 6.5 & 0.02 & 0.6 & 0 . \\
\hline Vigor & 5.1 & \pm 4.1 & 7.1 & \pm 5.5 & 6.7 & \pm 3.7 & 7.4 & \pm 4.0 & 0.7 & 0.40 & 3.6 & 0.07 & 1.0 & $0 .:$ \\
\hline Fatigue & 10.3 & \pm 6.1 & 8.4 & \pm 5.7 & 8.4 & \pm 5.0 & 7.5 & \pm 5.1 & 0.9 & 0.35 & 3.4 & 0.07 & 0.5 & 0. \\
\hline Confusion & 8.7 & \pm 3.9 & 5.8 & \pm 3.3 & 6.0 & \pm 3.1 & 5.6 & \pm 2.8 & 3.0 & 0.09 & 10.1 & 0.003 & 6.5 & 0.1 \\
\hline TMD & 34.2 & \pm 22.4 & 23.3 & \pm 19.4 & 24.2 & \pm 18.9 & 18.4 & \pm 16.8 & 2.1 & 0.15 & 10.2 & 0.003 & 0.9 & $0 .:$ \\
\hline \multicolumn{15}{|l|}{ SF-8 } \\
\hline PCS & 38.9 & \pm 8.2 & 44.3 & \pm 8.0 & 37.2 & \pm 9.1 & 46.0 & \pm 6.8 & 0.0 & 0.98 & 27.0 & $<0.0001$ & 1.5 & $0 .:$ \\
\hline MCS & 40.4 & \pm 8.4 & 44.0 & \pm 9.4 & 43.4 & \pm 10.2 & 45.3 & \pm 5.6 & 1.0 & 0.32 & 4.2 & 0.047 & 0.5 & 0. \\
\hline \multicolumn{15}{|l|}{ LNG } \\
\hline $\begin{array}{l}\text { Eye- } \\
\text { opening }\end{array}$ & 105.6 & \pm 36.3 & 97.4 & \pm 29.3 & 101.6 & \pm 38.9 & 94.7 & \pm 34.8 & 0.1 & 0.72 & 2.5 & 0.12 & 0.0 & 0.1 \\
\hline $\begin{array}{l}\text { Eye- } \\
\text { closing }\end{array}$ & 151.4 & \pm 56.1 & 120.4 & \pm 46.4 & 176.4 & \pm 116.9 & 137.2 & \pm 51.4 & 1.3 & 0.25 & 9.3 & 0.004 & 0.1 & 0. \\
\hline \multicolumn{15}{|l|}{ ENV } \\
\hline $\begin{array}{l}\text { Eye- } \\
\text { opening }\end{array}$ & 8.4 & \pm 5.5 & 6.8 & \pm 6.1 & 6.1 & \pm 6.5 & 4.8 & \pm 3.3 & 2.3 & 0.14 & 2.8 & 0.10 & 0.0 & 0.1 \\
\hline $\begin{array}{l}\text { Eye- } \\
\text { closing }\end{array}$ & 10.9 & \pm 7.4 & 7.3 & \pm 5.5 & 10.5 & \pm 12.7 & 7.1 & \pm 5.4 & 0.0 & 0.87 & 6.0 & 0.02 & 0.0 & 0.1 \\
\hline
\end{tabular}

* VM > VN at time $1(F=6.8, p=0.01)$; time $1>$ time 2 in VM $(F=17.6, p<0.0001)$

VM (vestibular migraine)

VN (vestibular neuritis)

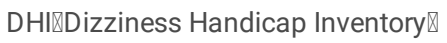

STAI (State-Trait Anxiety Inventory)

SDS $₫$ Self-Rating Depression Scale)

POMS (Profile of Mood Status)

TMD (total mood disturbance)

SF-8 (short form 8)

PCS (physical component score)

MCS (mental component score)

LNG (the total length of path) 
EVN (environmental area)

Page 7/7 